\title{
Research on Latent Semantic Model and user model-based video recommendation Algorithm
}

\author{
FuHao-Yang ${ }^{1, a^{*}, \text { ShaoFu-Lin }}{ }^{2, b}$ \\ ${ }^{1}$ Beijing University of Technology, Beijing China \\ ${ }^{2}$ Beijing University of Technology, Beijing China \\ a Igeling27@163.com, b linshaofu@bjut.edu.cn
}

Keywords: Video recommendation; Latent semantic model; Neighborhood method; Fusion.

\begin{abstract}
The rapid progress of Internet technology brings new opportunities for the development of science and technology, and the emerging network video technology is gradually penetrated into people's daily life. Due to the rapid development, the plentiful video contents also make everyone dazzling, at the same time, the video users with multiple geometric growth also make the network video operators not know what to do, and the fundamental technology to solve this problem is video recommendation technology. This paper presents an algorithm combined with the neighborhood latent semantic model, the new model retains the characteristics of recommended explanation in neighborhood algorithm, and expands based on implicit feedback information of users, which has further improved the recommendation efficiency. The new model adopts the field method of User-CF, this paper will compare the operational effects of basis latent semantic and the latent semantic fusing User-CF neighborhood mode to achieve the purpose of simulation.
\end{abstract}

\section{Introduction}

The existence of the information age has its certain inevitability, and the transmission of scientific and technological information is also beneficial to the progress of human civilization. In many developed countries and even some developing countries, they attach great importance to the national informatization, and consider the development direction and strategy of the national informatization from the overall strategic point of view [1]. As one of the main carriers of recording information, videos are vivid and easy to understand, they spread widely in daily life and influence greatly. Now the network technology is relatively mature, there have been many video resources sharing platforms, and the number of video resources has increased rapidly [2,3]. A simple video recommendation software will greatly shorten the time we spend in searching our favorite videos.

Market value created by rapidly developing network video industry is also high, for example, in 2015, China's online video industry has reached the market scale of 2.42 billion yuan, with a year-on-year growth of $31.5 \%$ [4]. In 2016, the single market advertising revenue of China Network Video had reached 9.02 billion yuan, which contributed to form a sustained and stable development situation, compared with the income in 2011, it had increased by $83.2 \%$ [5].

The above survey data show the number of video, the volume of users have increased dramatically. Changes in mobile video and online video allow ordinary video users to enjoy entertainment that was unimaginable in the past, readily available video resources make users no longer bothered about lack of video resources, but the video content becomes too busy to attend to all, and to solve this problem, we must rely on mature recommendation techniques and effective applications [6,7]. And the promotion of user experience will become the core of competition for network video operators. In order to enhance competitiveness, enterprises must provide effective personalized recommendation service for users. Operators can no longer simply provide users with the most hottest or most popular resources, otherwise it will lead to a low user experience and then drain customers. Users' behavior history will become the focus of video operators' mining and analysis, to provide users with interesting and suitable resources, we need to analyze user interests systematically, only in this way can we maintain user loyalty and 
viscosity [8,9]. In this process, we should also maximize the use of our own massive resources, and then get the maximum viewing rate and economic value.

Normally, there are three main reasons for users to get access to video websites: searching known videos from other sources, or searching videos related to the topic of concern, and simply pursuing entertainment to search videos of interest. The solution of video recommendation technology is to prepare for the last scenario, recommendation system can provide personalized selection, so as to help users to easily discover high quality videos [10]. At the same time regularly updated recommendation can maintain the user's activity and satisfaction, and record users' website activities.

Personalized video recommendation technology has been applied in the Internet and TV programs in recent years, and the effect is very good. A typical example is YouTube, they succeeded in practicing the success of the item based recommendation algorithm, and came to such conclusion that user click rate on Personalized Recommendation video is twice as that of common popular videos. However, this recommendation technology has high limitations, and it can not make full use of and mining information of users and goods, and it needs to improve the quality and efficiency of recommendation [11].

At present, the collaborative filtering system is mainly used in video software to meet the needs of users. Collaborative filtering technology is to use the user's previous operating data to predict what might users like, so as to produce recommendations. In most cases, the user's demand for video resources is completely different, but usually we may see consistent film reviews, if there is a certain similarity in videos interested by users, then, the video software will seek the closest video to the user's preferences through collaborative filtering on the basis of the user's model, instead of relying on the original similarity to get the final video screening results, at the same time, the complexity and precision of this algorithm is much simpler than traditional domain computation, but the result is more accurate, such method is called latent semantic model [12]. Therefore, the paper will analyze the basic Latent Semantic and Latent Semantic fusing User-CF neighborhood mode, in order to finally achieve the purpose of simulation.

\section{Latent semantic model and Latent semantic model fusing User-CF neighborhood method}

Latent semantic model. In 2006, latent semantic model was first published by Simon Funk on the blog, and it was later called as Latent Factor Model by Koren, champion of Netflix Prize, that is, LFM, on the basis of SVD decomposition, the singular matrix in the center is separated and incorporated into the singular matrix on the left and right sides, the whole operation steps are as follows:

$$
R=(U S) V^{r}=U\left(S V^{r}\right)=(U \sqrt{S})\left(\sqrt{S} V^{r}\right)
$$

After combining the items in parentheses: organize them as:

$$
R=U V^{r}
$$

Wherein, $\mathrm{U}$ represents the user characteristic matrix, and $\mathrm{V}$ represents the item characteristic matrix. Using the same dimensionality reduction method as SVD, that is, the maximum singular value of one $\mathrm{f}$ is used to reduce the dimension of matrix $R$, then it is divided into two multiplied low dimensional singular matrices:

$$
\hat{R}=P Q^{t}
$$

In the formula, $\mathrm{P}$ is a $m \times f$ order matrix, it marks the potential connections between users and prominent features, and $\mathrm{Q}$ is a $n \times f$ matrix, it represents the potential link between the material resources and the prominent features. Fig. 1 is a schematic diagram of the LFM decomposition. 


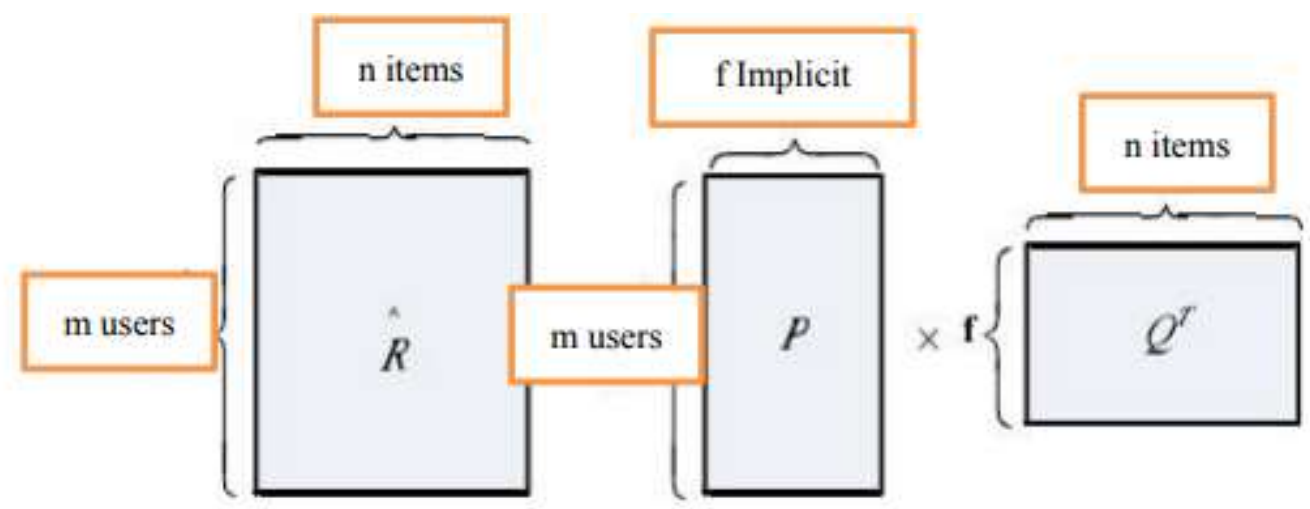

Figure 1. Schematic diagram of LFM decomposition

LFM predicts the correlation between the user $\mathrm{u}$ and the item $\mathrm{i}$ through the following calculation formula:

$$
\hat{r}_{u i}=p_{u} q_{i}^{T}=\sum_{f=1}^{F} p_{u, f} q_{i, f}
$$

$p_{u, f}$ and $q_{i, f}$ act as the parameters of the model, $p_{u, f}$ means the relationship between user's $\mathrm{u}$ concerns and implicit features (the $\mathrm{f}$ one), that is, the degree of interest of the user $u$ to the $\mathrm{f}$ implicit class; $q_{i, f}$ means the relationship between item i and f implicit feature, it also describes the weight of item $\mathrm{i}$ proportion on the implicit feature of $\mathrm{f}$.

According to the LFM model, it is found that LFM model can be used to predict the user's interest in objects in collaborative filtering, it has following two reasons: 1, The establishment of LFM model is to communicate users and videos with hidden classes; 2, at first, LFM was introduced into collaborative filtering algorithm to reduce dimension, so the problem of data sparsity and scalability can be solved by this method.

Solution of Latent semantic model. As there is LFM mode, the next problem is the calculation of the two parameters $p_{u, f}$ and $q_{i, f}$. In order to obtain this parameter, the first step is to have a set of training items that cover the interested items by the user based on optimization and machine learning theory, and the model parameters depend on the data learning in this data set. Suppose $K=\{(u, i)\}$, wherein, $(u, i)$ means single sample, and once $u$ had an action on the object $\mathrm{i} ; \hat{r}_{u i}$ means the prediction interest of user $\mathrm{u}$ to object $\mathrm{i}, r_{u i}$ means the actual interest of user $\mathrm{u}$ to object $\mathrm{i}$; the square of user interest error can be obtained by the following publicity:

$$
C=\sum_{(u, i) \in K}\left(r_{u i}-\hat{r}_{u i}\right)^{2}=\sum_{(u, i) \in K}\left(r_{u i}-\sum_{f=1}^{F} p_{u, f} q_{u, f}\right)^{2}
$$

On analysis of the above formula, it is visible that we need to optimize the data set as users have already done on the item, at this time, the calculation quantity is less, and it avoids the loss of score values in matrix completion.

Finding the optimal form of matrix decomposition is the goal of LFM algorithm, thus, the prediction matrix $\hat{R}$ and the training matrix $\mathrm{K}$ obtained by the decomposition can be same as much as possible, at the same time, the corresponding values of the blank items in the original matrix in the $\hat{R}$ and the test set will be the same as possible. Therefore, minimizing the sum of squares of interest errors becomes the optimal solution of the matrix decomposition, and the sum of squares is the loss function in the above formula.

But because of the sparsity of data, over fitting problem will occur. In order to avoid this problem, it is necessary to add regularization term to the above formula to avoid over fitting, as shown below: 


$$
C=\sum_{(u, i) \in K}\left(r_{u i}-\sum_{f=1}^{F} p_{u, f} q_{u, f}\right)^{2}+\lambda\left(\left\|p_{u}\right\|^{2}+\left\|q_{i}\right\|^{2}\right)
$$

Wherein, $\lambda$ is a regularization parameter obtained by simulation. In order to obtain the minimum value of the loss function in the upper formula, it is necessary to apply the method of optimization theory, and the stochastic gradient descent algorithm is the most basic method in this optimization method. Firstly, the partial derivative of the parameter is found, so that the parameter whose function value drops fastest can be found, then, using iterative method to find the parameters when the loss function is optimal. Conduct partial derivative to $p_{u, f}$ and $q_{i, f}$, the results as:

$$
\frac{\partial C}{\partial p_{u, f}}=-2\left(r_{u i}-\sum_{f=1}^{F} p_{u, f} q_{i, f}\right) q_{i, f}+2 \lambda p_{u, f}
$$

In this way, the direction of steepest descent of the parameter can be obtained, and the following recurrence formula can be obtained by advancing in this direction:

$$
\begin{aligned}
& p_{u, f}=p_{u, f}+\alpha\left[\left(r_{u, i}-\sum_{f=1}^{F} p_{u, f} q_{i, f}\right) q_{i, f}-\lambda p_{u, f}\right] \\
& q_{u, f}=q_{u, f}+\alpha\left[\left(r_{u, i}-\sum_{f=1}^{F} p_{u, f} q_{i, f}\right) p_{i, f}-\lambda q_{u, f}\right]
\end{aligned}
$$

Wherein, $\alpha$ means learning rate, it is usually gained by experiment. In order to converge the algorithm as fast as possible, the value needs to be attenuated every time the iteration is done, and the usual attenuation factor is taken as 0.9 . Before starting the parameter learning process, it is necessary to initialize the feature matrix $\mathrm{P}$ and $\mathrm{Q}$. In a variety of initialization methods, the random number filling method is the most useful. According to the experience, this random number is inversely proportional to the square root of the number of hidden classes $F$.

Latent semantic model fusing User-CF neighborhood method. The latent semantic model with User-CF introduced is replaced by U-LFM in this paper. First, we can get the learning model of User-CF:

$$
\hat{r}_{u i}=\frac{1}{\sqrt{\left|N_{i}\right|}} \sum_{v \in N_{i}} w(u, v)
$$

Wherein, $N_{i}$ means the set of all users like item $\mathrm{i}$.

Then, split $w(u, v)$ into two lower dimensional vectors $p_{u}$ and $z_{v}$, they are vectors in two directions of $\mathrm{F}$ dimension, that is, substitute $w(u, v)=p_{u} z_{v}{ }^{T}$ into above formula to gain:

$$
\hat{r}_{u i}=\frac{1}{\sqrt{\left|N_{i}\right|}} \sum_{v \in N_{i}} p_{u} z_{v}{ }^{T}=\frac{1}{\sqrt{\left|N_{i}\right|}} p_{u} \sum_{v \in N_{i}} z_{v}{ }^{T}
$$

By calculating the learning model of User-CF and combining it with the LFM model, the following new numerical models are obtained, that is, U-LFM:

$$
\hat{r}_{u i}=p_{u} q^{r}{ }_{i}+\frac{1}{\sqrt{\left|N_{i}\right|}} p_{u} \sum_{v \in N_{i}} z_{v}{ }^{T}=p_{u}\left(q^{r}{ }_{i}+\sum_{v \in N_{i}} z_{v}{ }^{T}\right)
$$

The biggest advantage of U-LFM compared with User-CF is to reduce the three-dimensional complexity of offline computing.

\section{Experimental Results and Evaluation}

This chapter uses the movie score data of MovieLens100K and the movie information dataset of u.item, wherein, u1.base exists in the role of training set. And U1.test exists in the role of the test set. In the process of simulation, the collected data sets are gradually input into the data model established in the 
paper, and on this basis, the LFM and U-LFM are computed respectively, finally, the advantages of offline simulation are used to evaluate the performance of both.

The data simulation for this chapter will be carried out independently for 10 times, and the results will be averaged. There are 4 parameters in latent semantic model: The number of hidden classes $F$, regularization parameters $\lambda$, learning rates $\alpha$, and the number of iterations $S$, the most critical data are regularization parameter $\lambda$, learning rate $\alpha$. Because the accuracy of the data is the ultimate goal of offline evaluation, therefore, the following two important simulation models are adopted to judge the degree of influence of accuracy of recommended arithmetic results of these two parameters on the basis of latent semantic model, and, a comprehensive comparative analysis is carried out in this regard.

The influence of regularization parameter $\lambda$.

Table 1 The accuracy rate of two kinds of latent semantic models under different values $\lambda$

\begin{tabular}{|c|c|c|c|c|c|c|}
\hline$\lambda$ & 0.01 & 0.015 & 0.02 & 0.025 & 0.03 & 0.035 \\
\hline LFM & 22.34 & 22.84 & 22.85 & 24.26 & 23.02 & 23.12 \\
\hline U-LFM & 22.82 & 22.94 & 23.15 & 24.35 & 23.54 & 23.31 \\
\hline
\end{tabular}

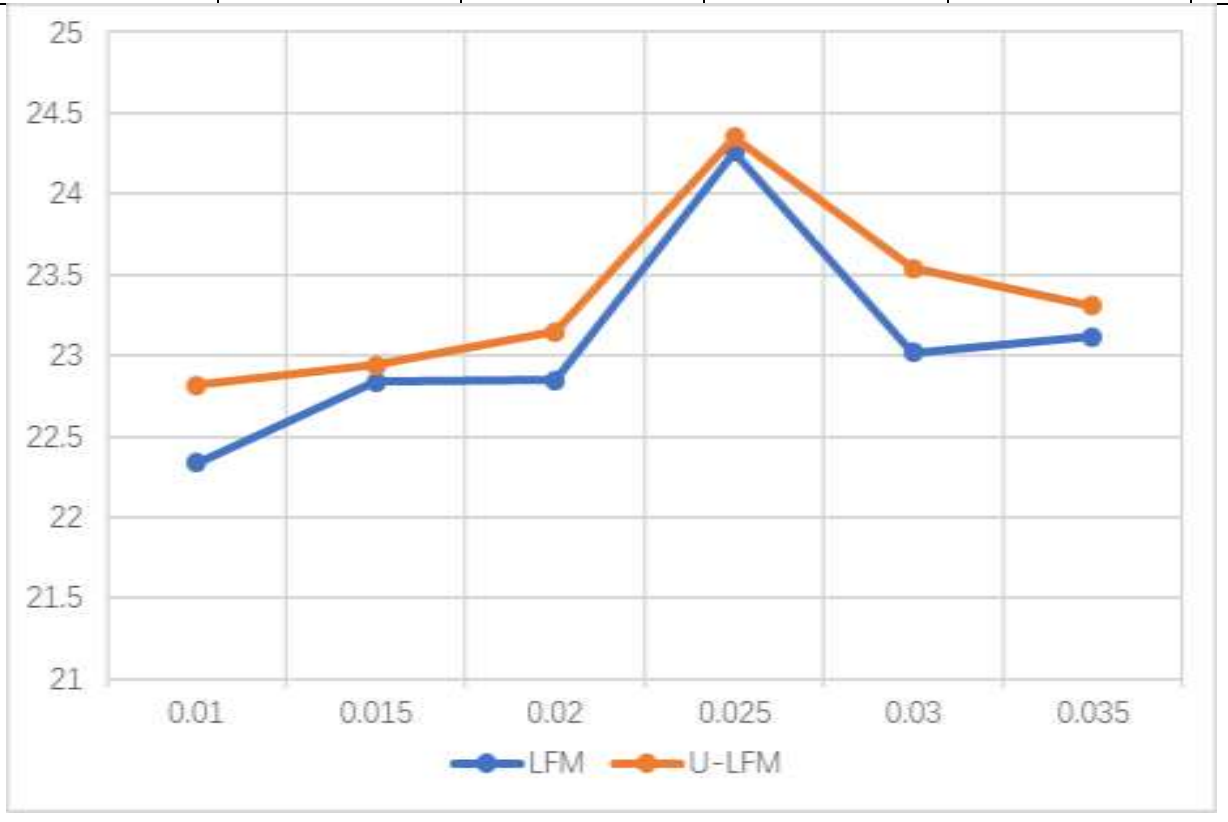

Figure 2. Accuracy of LFM and U-LFM models at different values $\lambda$

The values $F=10, \alpha=0.02, S=10$ are assigned respectively, and then the constant values are used to obtain the results.

The simulation results are shown in Table 1 , when $\lambda=0.025$, LFM and U-LFM haave the highest accuracy, and that of latter is almost $0.14 \%$.

From the above figure, we can intuitively draw the trend of the two broken lines, all show the trend of rising first and falling later, this is the same as the actual situation: the existence of $\lambda$ is to prevent the occurrence of false data caused by the over fitting of two curves. The greater the numerical value, the worse the fitting effect, the more inaccurate the result, otherwise, it will be not referential.

The influence of learning rate $\alpha$. The values $F=10, S=10$ are assigned respectively, $\lambda$ value when the accuracy rate is the best is assigned to both two algorithms, that is, LFM and U-LFM are assigned with $\lambda=0.025$. Finally, the relationship between learning rate $\alpha$ and accuracy of recommendation results is discussed.

As shown in Table 2 when $\alpha=0.015$, the calculated accuracy is the best. According to the accuracy predicted in the table, a is used as abscissa, and the accuracy is ordinate, in this way, $\mathrm{i}$ can simulate the performance reference line of LFM and U-LFM algorithms, as shown in Fig. 3. 
Table 2 Accuracy of LFM and U-LFM models at different $\alpha$ values

\begin{tabular}{|c|c|c|c|c|}
\hline$\alpha$ & 0.01 & 0.015 & 0.02 & 0.025 \\
\hline LFM & 22.04 & 23.12 & 23.21 & 22.86 \\
\hline U-LFM & 21.93 & 23.21 & 23.32 & 22.73 \\
\hline
\end{tabular}

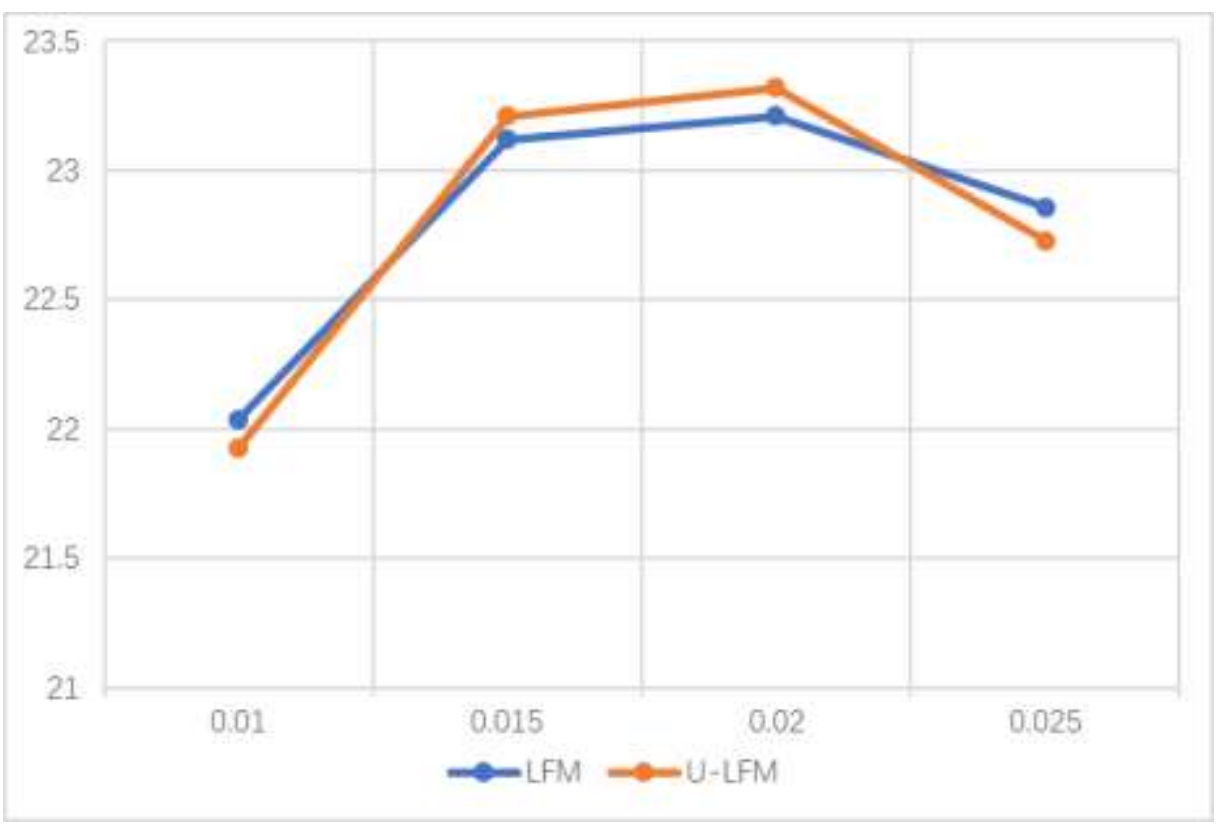

Figure 3. Accuracy of LFM and U-LFM models at different $\alpha$ values

The improved model is more effective in improving the accuracy rate, there are different degrees of performance at different learning rates, even when $\alpha$ is 0.01 , there's still a good rate of accuracy, this proves that the recommendation performance is still relatively good even when the initial search range is small. When $\alpha$ is 0.02 , the accuracy of the two models is the highest, and the U-LFM is improved by $0.47 \%$.

\section{Summary}

Based on two simulations, it is found in this paper that the recommendation effect of latent semantic model fusing User-CF neighborhood method has been definitely improved compared to that of the original latent semantic model, therefore, it can be popularized in practical application.

\section{References}

[1] H Koohi, K Kiani. “User Based Collaborative Filtering using Fuzzy CMeans,"Measurement. Vol. A91, pp. 134-139, May 2016.

[2] C Chen, H Yin, J Yao, B Cui. "TeRec: a temporal recommender system over tweet stream," Proceedings of the Vldb Endowment. Trento, vol.A6, pp. 1254-1257, August 2013.

[3] Y Ar, E Bostanci. "A genetic algorithm solution to the collaborative filtering problem," Expert Systems with Applications. Vol. A61, pp.122-128, May 2016.

[4] Z Liu. "Collaborative Filtering Recommendation Algorithm Based on User Interests," International Journal of u- and e- Service, Science and Technology. Suining, vol. A8, pp. 311-320, April 2015.

[5] W Cheng, G Yin, Y Dong, H Dong, W Zhang. "Collaborative Filtering Recommendation on Users' Interest Sequences,” Plos One. Harbin, vol.A11, pp. 1-17, May 2016. 
[6] Y Xiao, A I Pengqiang, C H Hsu, H Wang, X Jiao. "Time-Ordered Collaborative Filtering for News Recommendation," Mobile Information Systems.Beijing, vol. A12, pp. 53-62, December 2015 .

[7] XIONG Jianying.Study on Trust Model of C2C E-Commerce Based on Feedback[D]. PhD thesis, Finance and Economics University Of Jiangxi,2013.

[8] Xie L, Jia X, Zhou K. QoS Multicast Routing in Cognitive Radio Ad Hoc Networks. International Journal of Communication Systems, 2012,25(1): 30-46.

[9] Hoque M, Hong X. BioStaR: A Bio-inspired Stable Routing for Cognitive Radio Networks. Proceedings of 2012 International Conference on Computing,Networking and Communications. Washington: IEEE Computer Society,2012.402-406.

[10] GAN Zao-Bin, DING Qian, LI Kai, XIAO Guo-Qiang.Reputation-Based Multi-Dimensional Trust Algorithm[J]. Journal of Software,2011,22(10):24012411.

[11] CHEN Xicun, ZHOU Huan, PIAO Wen.Analysis of eBays virtual terminal network[J]. Journal of Liaoning Technical University(Social Science Edition),2010(12).

[12] Wang Y,Vassileva J. Bayesian network-based trust model[C]//Web Intelligence,2003. WI2003. Proceedings. IEEE, IEEE, 2003: 372-378. 